\title{
COMPUTATIONAL APPROACH TO STEADY-STATE EUTECTIC GROWTH
}

\author{
David A. KESSLER \\ Department of Physics, University of Michigan, Ann Arbor, Michigan 48109, USA
}

and

\section{Herbert LEVINE}

Department of Physics and Institute for Nonlinear Science, University of California at San Diego, La Jolla, California 92093, USA

Received 15 May 1988; manuscript received in final form 18 October 1988

We formulate a boundary integral approach to the determination of periodic steady-state eutectic growth patterns. The numerical implementation of this method allows us to compute the band of allowed velocities for a given undercooling. We illustrate how this works in a simplified symmetric eutectic solidifying exactly at the eutectic composition.

\section{Introduction}

Constant velocity lamellar eutectic patterns are observed during free growth sufficiently close to the eutectic composition [1]. The determinant of the allowed wavelengths and velocities is important both for our understanding of the general problem of pattern formation in non-equilibrium systems as well as the practical control of alloy microstructure. Also, a careful study of this problem is the first step towards formulating a more complete theory of the full range of possible eutectic structures.

There have been many previous studies of lamellar eutectics [1]. Perhaps the best known is the work of Hunt and Jackson [2] who solved the equation analytically by employing several approximations. Later, Dayte and Langer [3] extended this work to include a discussion of possible instabilities at off-eutectic compositions. These works made significant progress towards elucidating the possible behavior, but had to make use of hard to justify assumptions to carry through the analysis.

In this paper, we develop and apply the boundary integral methodology for this system.
That is, we rewrite the equation of motion as an integro-differential equation for the interface position. This technique has proven to be very powerful in earlier numerical studies [4,5] of steady-state patterns in directional solidification. It is significantly more efficient than methods which solve the field equations over the entire region by, say, finite element decomposition. Here, we use a similar approach for eutectic growth, and present some sample calculations on a simplified version of the full problem.

This paper contains several important results. First, we demonstrate the ease with which one can find an explicit solution for the band of allowed patterns at fixed undercooling. These solutions correspond to steady-state lamellar structures of varying velocity and wavelength. Next, we show that there is indeed a maximum velocity at fixed undercooling, as indicated in the approximate analytical calculations of Hunt and Jackson. Finally, we find a fold in the solution branch, corresponding to a maximum value of $p=v \lambda / 4 D$ for velocity $v$, wavelength $\lambda$ and diffusion constant $D$. The velocity at this fold increases as $\Delta^{2}$ for undercooling $\Delta$. We compare this to a related finding in directional solidification. 
The outline of this paper is as follows. In section 2, we review the derivation of an integrodifferential equation, employing standard Green function techniques to the diffusion equation. In section 3, we describe our numerical algorithm for generating solutions. Section 4 presents our results for the symmetric eutectic solidifying at the eutectic composition. Finally, section 5 describes extensions to this study currently in progress.

\section{Integro-differential equation}

Let us start with a standard eutectic phase diagram [1] such as that shown in fig. 1. The slopes of the solidus and liquidus lines for the A-rich and B-rich phases are respectively denoted by $m_{\mathrm{s}}^{\mathrm{A}, \mathrm{B}}, m_{\ell}^{\mathrm{A}, \mathrm{B}}$. The eutectic composition, denoted by $C_{\mathrm{E}}$, is the point at which the two liquidus lines meet, at the eutectic temperature $T_{\mathrm{E}}$.

The equations describing eutectic crystallization are as follows. First, the concentration in each phase satisfies the diffusion equation

$D_{\mathrm{i}} \nabla^{2} C=\partial C / \partial t$,

where the diffusion constants are phase dependent. At large distances from the front, $C \rightarrow C_{\infty}$. Next, conservation of matter gives rise to the Stefan boundary condition.

$\left.D_{\ell} \hat{n} \cdot \nabla C\right|_{\ell}-\left.D_{\mathrm{A}} \hat{n} \cdot \nabla C\right|_{\mathrm{s}}=-\left(C_{\ell}^{\mathrm{int}}-C_{\mathrm{s}}^{\mathrm{A}}\right) v_{\mathrm{n}}$,

$\left.D_{\ell} \hat{n} \cdot \nabla C\right|_{\ell}-\left.D_{\mathrm{B}} \hat{n} \cdot \nabla C\right|_{\mathrm{s}}=-\left(C_{\ell}^{\mathrm{int}}-C_{\mathrm{s}}^{\mathrm{B}}\right) v_{\mathrm{n}}$,

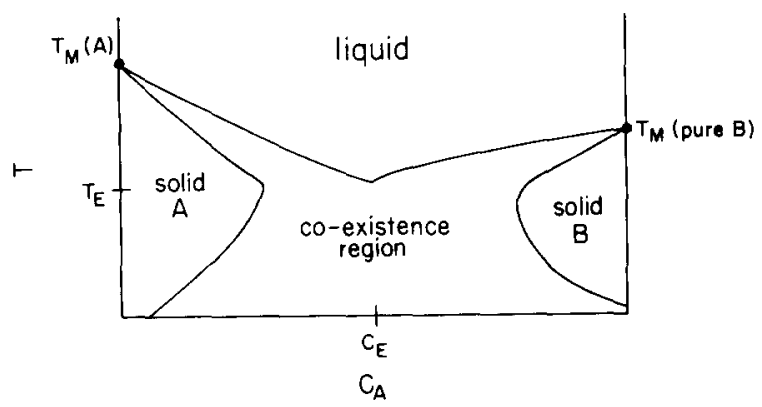

Fig. 1. Binary eutectic phase diagram. where $v_{\mathrm{n}}$ is the normal velocity and $C_{\ell}^{\mathrm{int}}, C_{\mathrm{s}}^{\mathrm{A}, \mathrm{B}}$ are the concentrations at the phase boundaries. The above two equations are valid at the liquid-solid A and liquid-solid B lines respectively.

We assume local thermodynamic equilibrium at a fixed temperature $T=T_{\mathrm{E}}-\tilde{\Delta}$. Using the Gibbs-Thomson condition, we derive

$m_{\ell}^{\mathrm{A}}\left(C_{\ell}^{\mathrm{int}}-C_{\mathrm{E}}\right)=\tilde{\Delta}-\tilde{\gamma}_{\mathrm{A}} \kappa=m_{\mathrm{s}}^{\mathrm{A}}\left(C_{\mathrm{s}}^{\mathrm{A}}-C_{\mathrm{E}}^{\mathrm{A}}\right)$,

$m_{\ell}^{\mathrm{B}}\left(C_{\ell}^{\mathrm{int}}-C_{\mathrm{E}}\right)=\tilde{\Delta}-\tilde{\gamma}_{\mathrm{B}} \kappa=m_{\mathrm{s}}^{\mathrm{B}}\left(C_{\mathrm{s}}^{\mathrm{B}}-C_{\mathrm{E}}^{\mathrm{B}}\right)$,

where $C_{\mathrm{E}}^{\mathrm{A}, \mathrm{B}}$ are the solid compositions at the eutectic temperature, and $\tilde{\gamma}_{\mathrm{A} . \mathrm{B}}$ are the capillary lengths $\sigma_{\mathrm{A}, \mathrm{B}} T_{\mathrm{m}}^{\mathrm{A}, \mathrm{B}} / L$ where $\sigma_{\mathrm{A}, \mathrm{B}}$ are the surface energies of the solid $\mathrm{A}$-liquid and solid $\mathrm{B}$-liquid interfaces, $L$ is the latent heat, and the temperatures $T_{\mathrm{m}}$ refer to the pure phase melting points.

Finally, the triple phase point equilibrium gives rise to the conditions

$\sigma_{\mathrm{A}} \cos \theta_{\mathrm{A}}+\sigma_{\mathrm{B}} \cos \theta_{\mathrm{B}}=\sigma_{\mathrm{AB}}$,

$\sigma_{\mathrm{A}} \sin \theta_{\mathrm{A}}=-\sigma_{\mathrm{A}} \sin \theta_{\mathrm{B}}$

if $\theta$ is the angle between the liquid-solid interface and the (vertical) $\mathrm{AB}$ phase boundary, with surface energy $\sigma_{\mathrm{AB}}$. This set of equations then allows for the evaluation of the normal velocity, given the current values of the interface and field variables.

We wish to focus on steady-state growth at some velocity $v$. Let us define a new dimensionless concentration variable

$u=\frac{C-C_{\mathrm{E}}}{C_{\mathrm{E}}^{\mathrm{A}}-C_{\mathrm{E}}}, \quad u_{\infty}=\frac{C_{\infty}-C_{\mathrm{E}}}{C_{\mathrm{E}}^{\mathrm{A}}-C_{\mathrm{E}}}$.

Similarly, we define the dimensionless variables

$$
\begin{aligned}
\Delta^{\mathrm{A}, \mathrm{B}} & =\frac{\tilde{\Delta}}{m_{\ell}^{\mathrm{A}, \mathrm{B}}\left(C_{\mathrm{E}}^{\mathrm{A}}-C_{\mathrm{E}}\right)}, \\
\gamma^{\mathrm{A}, \mathrm{B}} & =\frac{v \tilde{\gamma}^{\mathrm{A}, \mathrm{B}}}{2 D_{\ell} m_{\ell}^{\mathrm{A}}, \mathrm{B}}\left(C_{\mathrm{E}}^{\mathrm{A}}-C_{\mathrm{E}}\right)^{-1} .
\end{aligned}
$$

We also need various ratios

$$
k^{\mathrm{A}, \mathrm{B}}=\frac{m_{\ell}^{\mathrm{A}, \mathrm{B}}}{m_{\mathrm{s}}^{\mathrm{A}, \mathrm{B}}}, \quad \alpha^{\mathrm{A}, \mathrm{B}}=\frac{D_{\mathrm{s}}^{\mathrm{A}, \mathrm{B}}}{D_{\ell}}, \quad \beta=\frac{C_{\mathrm{E}}^{\mathrm{B}}-C_{\mathrm{E}}}{C_{\mathrm{E}}^{\mathrm{A}}-C_{\mathrm{E}}} .
$$


Finally, we rescale all lengths by the diffusion length $v / 2 D_{\ell}$. Then the above equations take the form

$\nabla^{2} u+2 \frac{\partial u}{\partial y}=0 \quad$ liquid,

$\alpha^{\mathrm{A}, \mathrm{B}} \nabla^{2} u+2 \frac{\partial u}{\partial y}=0 \quad$ solid,

$u \rightarrow u_{\infty}$ as $x \rightarrow \infty$,

$\left.\hat{n} \cdot \nabla u\right|_{\ell}-\left.\alpha^{\mathrm{A} \cdot \mathrm{B}} \hat{n} \cdot \nabla u\right|_{\mathrm{s}}=-2\left(u_{\ell}^{\mathrm{A}, \mathrm{B}}-u_{\mathrm{s}}^{\mathrm{A} \cdot \mathrm{B}}\right)$.

where the values of the field at the interfaces are

$u_{\ell}^{\mathrm{A}}=\Delta^{\mathrm{A}}-\gamma^{\mathrm{A}} \kappa, \quad u_{\ell}^{\mathrm{B}}=\Delta^{\mathrm{B}}-\gamma^{\mathrm{B}} \kappa$,

$u_{\mathrm{s}}^{\mathrm{A}}=1+k^{\mathrm{A}}\left(\Delta-\gamma^{\mathrm{A}} \kappa\right), \quad u_{\mathrm{s}}^{\mathrm{B}}=\beta+k^{\mathrm{B}}\left(\Delta-\gamma^{\mathrm{B}} \kappa\right)$.

This derivation shows that the system is determined completely by the growth conditions $\Delta^{\mathrm{A}, \mathrm{B}}, u_{\infty}$, and the material parameters, $\gamma^{\mathrm{A}, \mathrm{B}}, k^{\mathrm{A}, \mathrm{B}}$, $\beta, \alpha^{\mathrm{A}, \mathrm{B}}$.

We can use by now familiar techniques to write the above system as an integrodifferential equation. Specifically, we construct the field $u_{1}\left(u_{2}, u_{3}\right)$ which is zero everywhere except in the liquid (solid A, solid B) region where it equals the actual field $u$. Let us label the liquid-solid $\mathrm{A}$ interface by $\Gamma_{\mathrm{A}}$, the liquid-solid $\mathrm{B}$ one by $\Gamma_{\mathrm{B}}$ and the vertical solid-solid line by $\Gamma_{\mathrm{AB}}$. Then, we can write down the integral equations

$$
\begin{aligned}
u_{1}= & u_{\infty}+\int_{\Gamma_{\mathrm{A}}}\left(\left(\hat{n}^{\prime} \cdot \nabla^{\prime} G\right) u_{\ell}^{\mathrm{A}}-G \phi\right) \\
& +\int_{\Gamma_{\mathrm{B}}}\left(\left(\hat{n}^{\prime} \cdot \nabla^{\prime} G\right) u_{\ell}^{\mathrm{B}}-G \phi\right), \\
u_{2}= & \int_{\Gamma_{\mathrm{A}}}\left(\left(\hat{n}^{\prime} \cdot \nabla^{\prime} G^{\mathrm{A}}\right) u_{\mathrm{s}}^{\mathrm{A}}+G^{\mathrm{A}} \phi^{\mathrm{A}}\right) \\
& +\int_{\Gamma_{\mathrm{AB}}}\left(\hat{n}^{\prime} \cdot \nabla^{\prime} G^{\mathrm{A}}\right) u_{\mathrm{s}}^{\mathrm{A}}, \\
u_{3}= & \int_{\Gamma_{\mathrm{B}}}\left(\left(\hat{n}^{\prime} \cdot \nabla^{\prime} G^{\mathrm{B}}\right) u_{\mathrm{s}}^{\mathrm{B}}+G^{\mathrm{B}} \phi^{\mathrm{B}}\right) \\
& +\int_{\Gamma_{\mathrm{AB}}}\left(\hat{n}^{\prime} \cdot \nabla^{\prime} G^{\mathrm{B}}\right) u_{\mathrm{s}}^{\mathrm{B}},
\end{aligned}
$$

where $\phi, \phi^{\mathrm{A}, \mathrm{B}}$ correspond to as yet unknown charge layers, and $G, G^{A, B}$ are the steady-state diffusive Green functions to be discussed in the next section.

Next, we must impose the Stefan condition (eq. (6)) on the general solution above; this yields

$$
\begin{aligned}
& \left(\phi-2 u_{\ell}^{\mathrm{A}, \mathrm{B}}\right)-\alpha^{\mathrm{A}, \mathrm{B}} \phi^{\mathrm{A}, \mathrm{B}}+2 u_{\mathrm{s}}^{\mathrm{A}, \mathrm{B}} \\
& =-2\left(u_{\ell}^{\mathrm{A}, \mathrm{B}}-u_{\mathrm{s}}^{\mathrm{A}, \mathrm{B}}\right) .
\end{aligned}
$$

This immediately implies

$$
\begin{array}{ll}
\phi=\alpha_{\mathrm{A}} \phi_{\mathrm{A}} & \text { (along } \left.\Gamma_{\mathrm{A}}\right), \\
\phi=\alpha_{\mathrm{B}} \phi_{\mathrm{B}} & \text { (along } \left.\Gamma_{\mathrm{B}}\right) .
\end{array}
$$

Note that we are not imposing the Stefan condition along the $\Gamma_{\mathrm{AB}}$ boundary, but instead are assuming that it always remains a straight vertical line. This corresponds to assuming that diffusion in either solid is much slower than in the liquid phase.

The final equations are then obtained by setting $u_{1}=0$ on the solid side of the $\Gamma_{\mathrm{A}}$ and $\Gamma_{\mathrm{B}}$ interfaces, and similarly setting $u_{2,3}=0$ on the liquid side of $\Gamma_{\mathrm{A}}$ and $\Gamma_{\mathrm{B}}$ respectively. Thus, at each point along the entire liquid-solid interface there are two equations to solve. The unknowns are the actual interface along $\Gamma_{\mathrm{A}}$ and $\Gamma_{\mathrm{B}}$ as well as $\phi$. In the next section, we describe a numerical procedure for obtaining solutions of these coupled equations.

There is one result that follows immediately from the above equation. Let us add all the equations and integrate over one wavelength with measure $\mathrm{d} x$. Then the only surviving term on the right hand side is the zeroth Fourier components of $G$ and $\hat{n} \cdot \nabla G$. We will soon derive explicit expression for these objects but for the moment, we note that

$$
\begin{aligned}
& (\hat{n} \cdot \nabla G)_{(0)}=0 \text { at } y=y^{\prime}, \\
& G_{(0)}=\alpha / 4 p,
\end{aligned}
$$

where $\alpha=1, \alpha^{\mathrm{A}}, \alpha^{\mathrm{B}}$ in the three phases respectively. Adding all the equations causes the G $\phi$ piece to cancel, leaving us with the result

$$
u_{\infty}\left(\lambda_{\mathrm{a}}+\lambda_{\mathrm{b}}\right)=\int_{\Gamma_{\mathrm{A}}} \mathrm{d} x^{\prime} u_{\mathrm{s}}^{\mathrm{A}}+\int_{\Gamma_{\mathrm{B}}} \mathrm{d} x^{\prime} u_{\mathrm{s}}^{\mathrm{B}}
$$


This just represents conservation of matter on the global scale. For the simple case $k^{\mathrm{A}}=k^{\mathrm{B}}=0$, $\beta=-1$, this reduces to the result

$C_{\infty}\left(\lambda_{\mathrm{a}}+\lambda_{\mathrm{b}}\right)=C_{\mathrm{s}}^{\mathrm{A}} \lambda_{\mathrm{a}}+C_{\mathrm{s}}^{\mathrm{B}} \lambda_{\mathrm{b}}$,

where $\lambda_{a}, \lambda_{b}$ are the widths of the $A$ and $B$ phases and the overall wavelength is $\lambda=\lambda_{\mathrm{a}}+\lambda_{\mathrm{b}}$.

\section{Numerical approach}

\subsection{Discretization}

To solve the previous set of equations, we need to consider several issues. First, we discuss the discretization of the interface, allowing us to replace the integro-differential equation by a set of coupled nonlinear algebraic equations. Next, we explain how to evaluate the Green functions (and derivatives thereof) appearing in the above expressions. Finally we describe the algorithm used to solve the resulting finite system.

Let us parameterize the interface as follows. We put points at equal arc length spacing $\mathrm{d} s_{\mathrm{A}}$ along $\Gamma_{\mathrm{A}}$ and $\mathrm{d} s_{\mathrm{B}}$ along $\Gamma_{\mathrm{B}}$. Let $i=0$ be the tip of phase A, $i=N$ the A-B boundary, $i=2 N$ the tip of phase B. We also define midpoints $j$ such that point $j$ is halfway between $i=j-1$ and $i=j$. In the interior of $\Gamma_{\mathrm{A}}, j$ runs from 1 to $N$ and in $\Gamma_{\mathrm{B}}$, from $N+1$ to $2 N$. Our dependent variables are taken to be the values $\theta_{j}$ of $\theta=\cos ^{-1}(\hat{n} \cdot \hat{y})$ at points $s_{j}$. In terms of $\theta_{j}$, the actual interface positions are given via

$x_{i}=x_{i-1}+\mathrm{d} s_{\mathrm{A}} \cos \theta_{j}, \quad y_{i}=y_{i-1}-\mathrm{d} s_{\mathrm{A}} \sin \theta_{j}$,

for $i=1, N$ and

$x_{i}=x_{i-1}+\mathrm{d} s_{\mathrm{B}} \cos \theta_{j}, \quad y_{i}=y_{i-1}-\mathrm{d} s_{\mathrm{B}} \sin \theta_{j}$,

for $i=N+1,2 N$. Note that $x_{0}=0$ by assumption and $y_{0}$ is also arbitrary and also set to zero. The total number of interface variables is $2 N+2$, counting $\mathrm{d} s_{\mathrm{A}}$ and $\mathrm{d} s_{\mathrm{B}}$. One relation between $\mathrm{d} s_{\mathrm{A}}$ and $\mathrm{d} s_{\mathrm{B}}$ can be determined by the requirement that the pattern has periodicity $\lambda$, that is via $x_{2 N}=\lambda / 2$; so, we can think of one of the unknowns as the wavelength. The other unknowns are $\phi_{i}, i=0, N-1$ and $i=N+1,2 N$. Note that $\phi$ is discontinuous at the triple phase point and its value approaching this point from either side is to be determined by extrapolation from the neighboring points. The total number of unknowns is $4 N+2$.

We therefore need to find $4 N+2$ equations. First, we have the integro-differential equations evaluated at the points $i=1, N-1$ and $i=N+1$, $2 N-1$, that is everywhere but the interval endpoints and the triple phase point. This gives us $2(2 N-2)$ equations. Next, the slope at the midphase points $i=0$ and $i=2 N$ as well as $\phi^{\prime}$ at the same two points must be zero. These give rise to the approximate conditions

$0=\frac{3}{2} \theta_{1}-\frac{1}{2} \theta_{2}=\frac{3}{2} \theta_{2 N}-\frac{1}{2} \theta_{2 N-1}$,

$0=\phi_{2}-4 \phi_{1}+3 \phi_{0}=\phi_{2 N-2}-4 \phi_{2 N-1}+3 \phi_{2 N}=0$.

This brings us to $4 N$ equations. Finally, we impose the triple phase equilibrium condition. This gives the two final equations involving,

$\theta_{\mathrm{A}}=\frac{3}{2} \theta_{N}-\frac{1}{2} \theta_{N-1}$,

$\theta_{\mathrm{B}}=\frac{3}{2} \theta_{N+1}-\frac{1}{2} \theta_{N+2}$,

which are just those given in eq. (4). This completes our description of the discretization procedure.

\subsection{Green function}

Let us now deal with the Green functions $G$, $G^{\mathrm{A}, \mathrm{B}}$. These satisfy

$\nabla^{2} G+\frac{2}{\alpha} \frac{\partial G}{\partial y}=\delta\left(x-x^{\prime}\right) \delta\left(y-y^{\prime}\right)$

with $\alpha=1$ in the liquid and $\alpha=\alpha^{\mathrm{A} . \mathrm{B}}$ in the solid A, B phase. A solution to this equation which satisfies the periodic boundary condition $\partial G / \partial x$ $=\left.0\right|_{x= \pm \lambda / 2}$ can be written as

$$
\begin{aligned}
G= & \frac{\alpha}{4 p} \mathrm{e}^{-\left(y-y^{\prime}+\left|y-y^{\prime}\right|\right) / \alpha}+\frac{\mathrm{e}^{-\left(y-y^{\prime}\right) / \alpha}}{2 p} \\
& \times \sum_{m}\left\{\cos \left(\frac{m \pi\left(x-x^{\prime}\right)}{p}\right)\right. \\
& \left.\times \exp \left(-\frac{1}{\alpha^{2}}+\frac{(m \pi)^{2}}{p}\left|y-y^{\prime}\right|\right)\right\} \\
& \times\left\{\sqrt{\frac{1}{\alpha^{2}}+\frac{(m \pi)^{2}}{p}}\right\}^{-1},
\end{aligned}
$$

where $p=\lambda v / 4 D$. 
The sum as it stands is not sufficiently convergent to be tractable numerically. We therefore proceed in one of two ways, depending upon whether $p / \alpha$ is small or large. For the former, we can do the sum exactly in the limit $\alpha \rightarrow \infty$. This then allows us to rewrite the above expression as

$$
\begin{aligned}
G= & \frac{\alpha}{4 p} \mathrm{e}^{-\left(y-y^{\prime}+\left|y-y^{\prime}\right|\right) / \alpha}-\frac{\mathrm{e}^{-\left(y-y^{\prime}\right) / \alpha}}{4 \pi} \\
& \times \log \left[1+\mathrm{e}^{-2 \pi\left|y-y^{\prime}\right| / p}-2 \cos \left(\frac{\pi\left(x-x^{\prime}\right)}{p}\right)\right. \\
& \left.\times \mathrm{e}^{-\pi\left|y-y^{\prime}\right| / p}\right] \\
& +\frac{\mathrm{e}^{-\left(y-y^{\prime}\right) / \alpha}}{2 p} \sum_{m} \cos \left(\frac{m \pi\left(x-x^{\prime}\right)}{p}\right) \\
& \times \frac{\exp \left(-\sqrt{\frac{1}{\alpha^{2}}+\left(\frac{m \pi}{p}\right)^{2}}\left|y-y^{\prime}\right|\right)}{\sqrt{\frac{1}{\alpha^{2}}+\left(\frac{m \pi}{p}\right)^{2}}} \\
& \left.-\frac{\exp \left(-m \pi\left|y-y^{\prime}\right| / p\right)}{m \pi / p}\right] .
\end{aligned}
$$

The sum is now absolutely convergent and can be evaluated to any desired accuracy by simple truncation.

A similar method works for $\hat{n}^{\prime} \cdot \nabla^{\prime} G$. If we apply the normal gradient to the above expression, the only new problem arises when the derivative acts on the terms inside the sum. These pieces have the form

$$
\begin{aligned}
& \frac{\mathrm{e}^{-\left(y-y^{\prime}\right) / \alpha}}{2 p}\left(\sum n_{x}^{\prime} \sin \left(\frac{m \pi\left(x-x^{\prime}\right)}{p}\right)\right. \\
& \times\left[\frac{\left.k_{x} \frac{\mathrm{e}^{-k_{y}\left|y-y^{\prime}\right|}}{k_{y}}-\mathrm{e}^{-k_{x}\left|y-y^{\prime}\right|}\right]}{}\right.
\end{aligned}
$$

$$
\begin{aligned}
& +\operatorname{sgn}\left(y-y^{\prime}\right) \sum n_{y}^{\prime} \cos \left(\frac{m \pi\left(x-x^{\prime}\right)}{p}\right) \\
& \left.\times\left[\mathrm{e}^{-k_{y}\left|y-y^{\prime}\right|}-\mathrm{e}^{-k_{x}\left|y-y^{\prime}\right|}\right]\right)
\end{aligned}
$$

with

$$
k_{x} \equiv \frac{m \pi}{p}, \quad k_{y}=\sqrt{\left(\frac{m \pi}{p}\right)^{2}+\frac{1}{\alpha^{2}}} .
$$

These sums need to be made more convergent, which can be accomplished via the replacement of these expressions with

$$
\begin{aligned}
& \frac{\mathrm{e}^{-\left(y-y^{\prime}\right) / \alpha}}{2 p}\left(\sum n_{x}^{\prime} \sin \left(\frac{m \pi\left(x-x^{\prime}\right)}{p}\right)\right. \\
& \times\left[\frac{k_{x} \mathrm{e}^{-k_{y}\left|y-y^{\prime}\right|}}{k_{y}}-\mathrm{e}^{-k_{x}\left|y-y^{\prime}\right|}\left(1+\frac{A}{k_{x}}\right)\right] \\
& +\operatorname{sgn}\left(y-y^{\prime}\right) \sum n_{y}^{\prime} \cos \left(\frac{m \pi\left(x-x^{\prime}\right)}{p}\right) \\
& \left.\times\left[\mathrm{e}^{-k_{y}\left|y-y^{\prime}\right|}-\mathrm{e}^{-k_{x}\left|y-y^{\prime}\right|}\left(1+\frac{A}{k_{x}}\right)\right]\right),
\end{aligned}
$$

with $A=-\left|y-y^{\prime}\right| / 2 \alpha^{2}$ This replacement then requires us to add to $\hat{n}^{\prime} \cdot \nabla^{\prime} G$ the additional term

$$
\begin{aligned}
& \frac{p A}{2 \pi} \mathrm{e}^{-\left(y-y^{\prime}\right) / \alpha} \\
& \times\left[n_{x}^{\prime} \tan ^{-1}\left(\frac{\mathrm{e}^{-\pi\left|y-y^{\prime}\right| / p} \sin \left(\frac{\pi\left(x-x^{\prime}\right)}{p}\right)}{1-\mathrm{e}^{-\pi\left|y-y^{\prime}\right| / p} \cos \left(\frac{\pi\left(x-x^{\prime}\right)}{p}\right)}\right)\right. \\
& +\pi y^{\prime} \operatorname{sgn}\left(y-y^{\prime}\right) \ln \left(1+\mathrm{e}^{-2 \pi\left|y-y^{\prime}\right| / p}\right. \\
& \left.\left.-2 \cos \left(\frac{\pi\left(x-x^{\prime}\right)}{p}\right) \mathrm{e}^{-\pi\left|y-y^{\prime}\right| / p}\right)\right]
\end{aligned}
$$

The final sums then are absolutely convergent and can be handled by truncation. 
In a recent paper, Karma [6] has suggested that in the small $p / \alpha$ limit, a useful approximation to $G$ can be obtained by dropping the final sum altogether. A similarly useful approximation to $\hat{n}^{\prime} \cdot \nabla^{\prime} G$ can be obtained by again dropping the final sum, but including the additional terms just computed. Once this is done, the error being made is uniformly of order $(p / \alpha)^{2}$ and can be safely neglected in many cases of interest.

For the case $p / \alpha$ large, the above method is not convenient. In particular, many terms in the sum will be necessary to achieve the desired accuracy. In this limit, it is more convenient to use the alternate expression

$$
\begin{aligned}
G= & \sum_{n=-\infty}^{\infty} \frac{\mathrm{e}^{-\left(y-y^{\prime}\right) / \alpha}}{\pi} \\
& \times K_{0}\left(\frac{1}{\alpha} \sqrt{\left(x-x^{\prime}+2 p n\right)^{2}+\left(y-y^{\prime}\right)^{2}}\right) .
\end{aligned}
$$

For $p / \alpha$ large, each successive term with higher $|n|$ will be exponentially suppressed relative to the $n=0$ piece. Derivatives of $G$ can be calculated by explicitly differentiating each term in the sum without any loss of convergence.

\subsection{The algebraic system}

The integrals in the evolution equation can be evaluated by the trapezoidal rule after explicitly dealing with any divergent integrands. As we have seen, there are singularities in both $G$ and $\hat{n}^{\prime} \cdot \nabla^{\prime} G$. After taking into account the $\delta$ function piece of $\hat{n}^{\prime} \cdot \nabla^{\prime} G$ by hand,

$\hat{n} \cdot \nabla^{\prime} G \sim \pm \frac{1}{2} \delta\left(s-s^{\prime}\right)$,

depending on whether $y \rightarrow y_{\text {int }} \pm \epsilon$, we have, at worst, logarithmically singular terms. These can be handled by explicit subtraction. For example, we replace $G \phi\left(s^{\prime}\right)$ by

$$
\begin{aligned}
& G\left(x, x^{\prime} ; y, y^{\prime}\right) \phi\left(s^{\prime}\right) \\
& \quad \rightarrow G\left(x, x^{\prime} ; y, y^{\prime}\right)\left[\phi\left(s^{\prime}\right)-\phi(s)\right] \\
& \quad-\frac{1}{4 \pi} \ln \left(s-s^{\prime}\right)^{2} \phi(s) .
\end{aligned}
$$

This then has a finite limit as $s \rightarrow s^{\prime}$. The last term can be integrated explicitly since $\phi(s)$ is independent of $s^{\prime}$. Similar subtractions work for the logarithmic divergences in $\hat{n}^{\prime} \cdot \nabla^{\prime} G$; as long as the factor multiplying the logarithm is evaluated at $s$ (as opposed to $s^{\prime}$ ) the subtraction terms can then be handled analytically.

Once this is done the trapezoidal rule gives the integral correct to $\mathrm{O}\left(1 / N^{2}\right)$. Derivatives (such as needed in the curvature $(\kappa=\partial \theta / \partial s))$ are evaluated by finite differences. The result of all this is a set of $4 N+2$ algebraic equations for the charge layer $\phi$ and the interface $\theta, \lambda$ and $\mathrm{d} s$. We also need to mention the integral over the AB vertical line. This integral converges exponentially as $y^{\prime} \rightarrow-\infty$ for $y$ fixed along either $\Gamma_{\mathrm{A}}$ or $\Gamma_{\mathrm{B}}$. In section 4 , for the special case $\beta=-1$, we will see how a slight shift of definition completely eliminates this term from the equations and we need not worry about this contribution at all.

We choose to solve this non-linear system by Newton's method. That is, we start with an initial guess for the solution and iterate towards a final set of values. The actual computation can be carried out using commercially available solvers, such as HYBRD from MINPACK or DZONE from PORT. Of course, the lack of convergence to a solution does not automatically mean that none exists; it is quite possible that a solution will be so far removed in the space of variables that an iterative procedure will be unable to find it. Nevertheless, our experience indicates that once one solution is found, nearby solutions (with small changes in, say, some of the experimentally tunable parameters) are quickly converged to; furthermore, if convergence fails it means that a solution branch has ended. We will see this explicitly in the example presented in section 4.

Before turning to a simple example of how the above algorithm works, we would like to comment on the generality of this approach. We have presented the formalism for the case of free eutectic growth, but it is obvious that changing to either directional solidification or eutectic directional solidification is quite easy. It is more difficult to extend everything to three dimensions but at least in the case of periodic structures, this too offers only some new technical details to be worked out. 
This methodology is much more powerful than the finite element methods that are currently being used. The basic reason for this is that all of our points lie on the interface, providing us with a detailed picture of the structure of the phase boundary. At the expense of a more complicated set of algebraic equation, we have eliminated the need to find the field throughout space, with a huge saving in computer time for a given interface resolution.

\section{A model calculation}

In this section we present numerical results on the simplest possible version of the general eutectic problem. We assume a completely symmetric eutectic solidifying at exactly the eutectic composition. In terms of our parameters

$u_{\infty}=0, \quad \beta=-1$,

$-\theta_{\mathrm{A}}=\theta_{\mathrm{B}}, \quad \Delta^{\mathrm{A}}=-\Delta^{\mathrm{B}} \equiv \Delta, \quad \gamma^{\mathrm{A}}=-\gamma^{\mathrm{B}} \equiv \gamma$.

Furthermore, we pick $\sigma_{\mathrm{AB}}$ so as to obtain $\theta_{\mathrm{A}}=\pi / 4$, $\alpha^{\mathrm{A}}=\alpha^{\mathrm{B}}=0$, and also $k^{\mathrm{A}}=k^{\mathrm{B}}=0$. The last conditions mean that the concentration is constant inside the solid phases, equaling 1 inside $A$ and -1 inside $\mathbf{B}$.

Starting from our basic equations, we can show that the condition $\hat{n} \cdot \nabla u_{2}=\hat{n} \cdot \nabla u_{3}=0$ inside the solid phases gives rise to $\phi=2 \hat{n}_{y} \epsilon^{\mathrm{A}, \mathrm{B}}$. Therefore, the final equation for the liquid region is

$0=\int\left(\hat{n}^{\prime} \cdot \nabla^{\prime} G\right)(\Delta-\gamma \kappa) \epsilon^{\mathrm{A}, \mathrm{B}}+\int 2 G \hat{n}_{y}^{\prime} \epsilon^{\mathrm{A}, \mathrm{B}}$,

where the factor $\epsilon^{\mathrm{A}, \mathrm{B}}=+1,-1$ in region $\mathrm{A}$ or $\mathrm{B}$. By symmetry, we can solve for the interface only in the region $i=0, N$ since $\theta_{i+N}=-\theta_{N-i}$. Similarly, choosing the full periodicity $\lambda$ means the position $x_{N}=\lambda / 4$; this then serves to fix $\mathrm{d} s_{\mathrm{A}}$.

The final variables are $\theta_{j}, j=1, N$ and $\lambda$ (or equivalently $p$ ), for a total of $N+1$. The integral equation is evaluated at $N-1$ points, $\theta_{\mathrm{A}}$ is fixed and $\theta(s=0)$ is zero. A solution of the equation is then found by solving these $N+1$ algebraic equations, at fixed $\gamma$ and $\Delta$. Once we have determined $p$ for a fixed input value of the parameter $\gamma$, we can work backwards from the defining formula

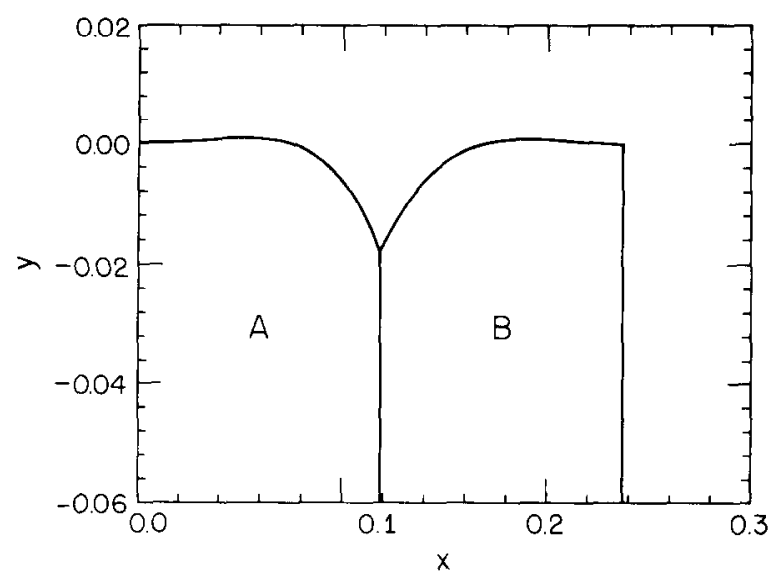

Fig. 2. Solution at $\Delta=0.1, p=0.06$.

$p=v \lambda / 4 D$ together with eq. (5b) to find the physical wavelength. Notice that the physical velocity of the solution is determined by $\gamma$ for fixed material parameters. This is similar to the situation which occurs in free dendritic growth where the only measure of surface energy effects is the (dimensionless) velocity.

We have studied $\Delta$ in the region from 0.05 to 0.25 , and solved for the shape and the velocity as a function al wavelength. A typical solution corresponding to $\Delta=0.1, p=0.0609$, and dimensionless velocity $3.65 \times 10^{-3}$ is shown in fig. 2 , In fig. 3 , we plot the band of allowed velocities at several

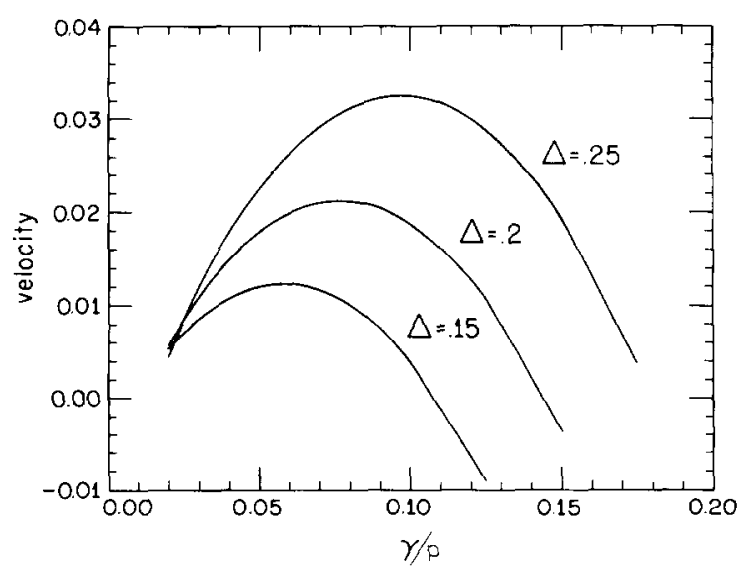

Fig. 3. Velocity band at $\Delta=0.15,0.20,0.25$. 

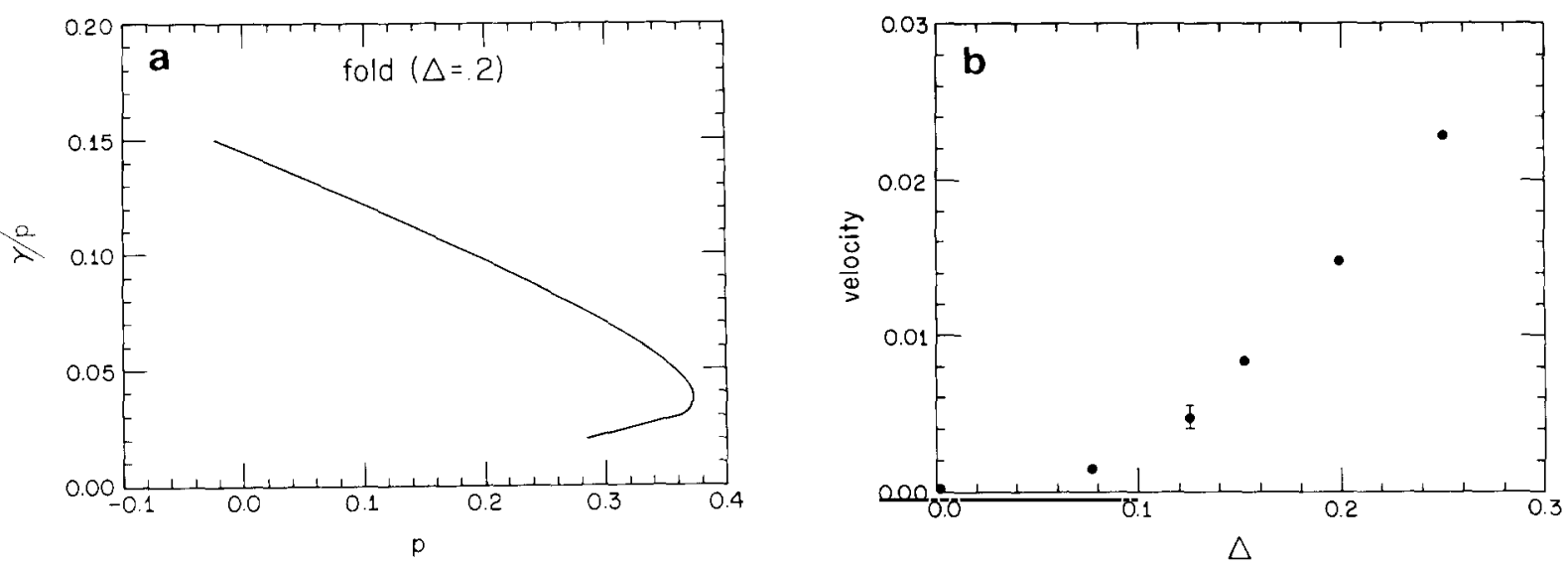

Fig. 4. Fold in $p$ : (a) $p$ versus $\gamma$ at $\Delta=0.2$; (b) velocity versus $\Delta$.

different undercoolings. In each case, there is a maximum allowed velocity. Early theories of pattern selection [7] suggested that this particular solution would be dynamically selected, but this assumption is completely ad hoc and is likely to be incorrect.

In fig. $4 \mathrm{a}$, we show the Peclet number $p=$ $v \lambda / 4 D$ along the solution branch corresponding to $\Delta=0.2$. We see that there is maximum possible value of $p$, which of course, does not coincide with the maximum velocity since the physical wavelength varies with velocity as we move through the band. The maximum corresponds to a fold in the solution branch when plotted versus $p$. This fold is a generic feature of many pattern forming systems. For example, a similar fold gives rise to a maximum Peclet number for cellular solutions at fixed drawing velocity during directional solidification [5].

In fig. $4 \mathrm{~b}$, we show the velocity corresponding to the solution at the fold, versus undercooling. The results seems to follow a $\Delta^{2}$ law. This scaling is consistent with most experimental studies [1]. One obvious hypothesis is that pattern selection may be connected to the existence of the fold. We have given [5] heuristic arguments that this might be the case for directional solidification; here, however, the situation is complicated by the coupling between velocity and wavelength selection.
Of course, only a complete dynamical theory can test whether this conjecture has any validity.

\section{Conclusions}

This paper has presented a straightforward but extremely powerful methodology for computing steady-state lamellar eutectic patterns. In principle, this method can be applied to any (two-dimensional) realistic system given knowledge of all the relevant thermodynamic parameters. Also, extension to three dimensions, while technically challenging, seems feasible.

To illustrate our ideas, we have studied the simplest possible model system, that of a completely symmetric eutectic with no diffusion in the solid and $k^{\mathrm{A}}=k^{\mathrm{B}}=0$. This system already exhibits some interesting structure, possibly related to the dynamical issue of velocity selection. Specifically, we showed that there is a maximum wavelength at any fixed undercooling due to a fold of the solution branch; this structure has been studied extensively in other systems and can be quite crucial in understanding steady-state structure.

There are clearly several areas which need to be worked on. First, we would like to take a specific realistic system and repeat the computations. We 
expect to find the same qualitative behavior as discussed here for a model problem but with a realistic system, we can compare our predicted band to actual experimental observations. Next, we would like to extend the steady-state methodology to the problem of linear stability. This would enable us to study various instabilities, either long wavelength or short, that have either been seen experimentally, predicted analytically or both.

Finally, we would like to use this methodology in an attempt to develop a theory of dynamical wavelength solution, possibly along the lines of what is knows to occur in convection or Taylor-Couette flow. This program of study, if successful and if extended to three dimensions, would in principle enable the systematic prediction of solidifcation microstructures during free eutectic growth, at least sufficiently close to the lamellar region.

\section{Acknowledgements}

The work of one of us (D.A.K.) was supported by US Department of Energy Grant No. DE-FG-
02-85ER54189. H.L. was supported in part by a grant from DARPA under University Research Initiative, Grant No. N00014-86-K-0758.

\section{References}

[1] For a review of the methodology of eutectic solidification as well as a guide to the vast metallurgical literature, see $\mathbf{R}$. Elliot, Eutectic Solidification Processing (Butterworths, London, 1983).

[2] K.A. Jackson and J.D. Hunt, Trans. Met. Soc. 236 (1966) 1129.

[3] V. Datye and J.S. Langer, Phys. Rev. B24 (1981) 4155.

[4] M. Ben-Amar and B. Moussallam, Phys. Rev. Letters 60 (1988) 317.

[5] D. Kessler and H. Levine, Cellular Patterns during Directional Solidification, Phys. Rev. A, to be published.

[6] A. Karma, Phys. Rev. Letters 57 (1986) 858.

[7] C. Zener, Trans. Met. Soc. 167 (1946) 550. 